\title{
Resveratrol induces MMP-9 and cell migration via the p38 kinase and PI-3K pathways in HT1080 human fibrosarcoma cells
}

\author{
EUN JEONG GWEON and SONG JA KIM \\ Department of Biological Sciences, College of Natural Sciences, Kongju National University, \\ Gongju, Chungnam 314-701, Republic of Korea
}

Received August 16, 2012; Accepted November 2, 2012

DOI: 10.3892/or.2012.2151

\begin{abstract}
Trans-3,4',5-trihydroxystilbene (resveratrol) is a grape polyphenol present in various plants, food products, red wine and grapes. Resveratrol has anti-inflammatory, anticarcinogenic, anti-oxidant and anti-aging properties. Matrix metalloproteinases (MMPs) are key enzymes involved in the degradation of the extracellular matrix, and their expression may be regulated in cancer metastasis. In the present study, we aimed to evaluate the effect of resveratrol on MMPs and cell migration, and to understand the mechanism of action in HT1080 human fibrosarcoma cells. We found that resveratrol inhibited HT1080 cell viability at various concentrations as detected by the MTT assay and FACS analysis. However, resveratrol dramatically increased the activation and expression of MMP-9 in a dose- and time-dependent manner, as determined by gelatin zymography assay and western blot analysis. We also discovered that resveratrol enhanced the migratory ability of HT1080 cells, as determined by the wound healing assay, and decreased the phosphorylation of p38 kinase. Moreover, the Akt kinase was inhibited by resveratrol in the HT1080 cells. The inhibition of p38 and Akt kinases with SB203580 and LY294002 further increased resveratrol-induced MMP-9 as well as cell migration in the HT1080 cells. Our results suggest that resveratrol regulates MMP-9 and migratory abilities through the p38 kinase and PI-3K pathways in HT1080 human fibrosarcoma cells.
\end{abstract}

\section{Introduction}

Trans-3,4',5-trihydroxystilbene (resveratrol) is a natural polyphenolic phytoalexin present in various plants, food products, red wine, grape skin, berries, nuts, eucalyptus and rheum. Its

Correspondence to: Professor Song Ja Kim, Department of Biological Sciences, College of Natural Sciences, Kongju National University, 182 Singwan-dong, Gongju, Chungnam 314-701, Republic of Korea

E-mail: ksj85@kongju.ac.kr

Key words: resveratrol, matrix metalloproteinase-9, migration, p38 kinase, phosphatidylinositol 3-kinase, HT1080 biological activities, including anti-inflammatory, anti-oxidant, anti-cancer, anti-aging, anti-bacterial, anti-fungal and anti-viral properties have been demonstrated (1). Resveratrol has been shown to prevent angiogenesis and the migration of endothelial cells, and this anti-angiogenic effect makes it a promising candidate for the prevention of cancer progression $(2,3)$. Resveratrol has been demonstrated to inhibit proliferation and induce apoptosis in gastric cancer cells. This provides the therapeutic rationale for tumor chemotherapy using resveratrol (4). Recent studies revealed that resveratrol inhibited cell invasion in hepatoma cells in response to hepatocyte growth factor in vitro as well as in hepatoma and Lewis lung carcinoma in mice (5). In addition, resveratrol was reported to suppress phorbol myristate acetate-induced cervical cancer cell invasion (6). Resveratrol may reduce proliferation, invasion, migration and apoptosis by regulating nuclear factor $(\mathrm{NF})-\kappa \mathrm{B}$ activities in various types of cancer cells (7). Noteworthy, resveratrol exerts biphasic effects where low concentrations are estrogenic and high concentrations are anti-estrogenic (8). High concentrations of resveratrol have anti-oxidant, pro-apoptotic, anti-growth, anti-angiogenic, anti-invasive and anti-inflammatory effects. Therefore, resveratrol is an important cancer-preventive agent (9). Another recent study found that resveratrol exhibits anti-cancer properties in different tumor cell types, including breast, prostate, stomach, colon, pancreatic and thyroid cancers (10).

Tumor metastasis occurs through a complex series of events including cell adhesion, migration, invasion, proliferation and vessel formation (11). Invasion and metastasis are fundamental properties of malignant cancer cells. The degradation of the basement membrane and stromal extracellular matrix (ECM), which exert biochemical and mechanical barriers to cell movement, has been demonstrated as an important biological process in the invasion and metastasis of cancer cells (12). ECM degradation and remodeling require the action of extracellular proteinases, among which matrix metalloproteinases (MMPs) have been noted to play a significant role. MMPs are a family of zinc-dependent proteinases involved in the degradation of the ECM. The MMPs have been implicated in the processes of tumor growth, invasion and metastasis. Frequently, overexpression of MMPs has been detected in malignant tumors and has been associated with an aggressive malignant phenotype and poor prognosis in patients with cancer (13). Among the MMPs, MMP-2 (72-kDa type IV collagenase) and MMP-9 (92-kDa type IV collagenase) have been reported to play a major role 
in cancer metastasis. It has been suggested that MMP-2 and MMP-9 are key mediators of tumor metastasis, migration and invasion among these members (14). Therefore, the inhibition of MMP expression and secretion is important to prevent cell adhesion, migration, invasion and metastasis. Moreover, MMP-2 and MMP-9 may be potential targets of anti-metastatic drugs for therapeutic benefit in cancer (15). The secretion and synthesis of MMPs may be promoted by a variety of stimuli, including cytokines, during various pathological processes, including tumor invasion, inflammation, atherosclerosis and rheumatoid arthritis (16). However, the mechanisms underlying the effect of resveratrol on MMPs and the migratory abilities in HT1080 human fibrosarcoma cells remain unclear.

Mitogen-activated protein kinase (MAPK) pathways are evolutionarily conserved kinase modules that link extracellular signals to the machinery that controls fundamental cellular processes, such as proliferation, growth, differentiation, migration, invasion and apoptosis in various cell types, including cancer cells (17). Abnormalities in MAPK signaling impinge on most, if not all of these processes and play a critical role in the progression and development of cancer (18). Recent studies have demonstrated the role of the p38 MAPK signaling pathway in mediating interleukin (IL)-28A-induced cell migration of UMUC-3 cells. The p38 pathway regulates IL-28A-induced cell migration through MMP-9 expression by activating NF- $\mathrm{B}$ and AP-1 binding motifs (19). Salinomycin exhibited significant growth inhibition and induction of apoptosis in the human ovarian cancer cell line OV2008. Effects of salinomycin on OV2008 cells have been associated with modulating the p38 MAPK signaling pathway. A previous study found that salinomycin-induced apoptosis in OV2008 cells may be associated with activating p38 MAPK and merits further investigation (20). Phosphatidylinositol 3-kinases (PI 3-kinases or PI-3Ks) are a family of enzymes involved in cellular functions, such as cell growth, proliferation, survival, differentiation, motility and intracellular trafficking. PI-3Ks are involved in various types of cancers. Links between PI-3K activity and several human maladies, including allergy, inflammation, heart disease and cancer have been noted. Noteworthy, various members of the PI-3K family have been linked to key cellular processes as well as to several human diseases (21). Recent studies have demonstrated that estrogen receptor $\beta$ growth-inhibitory effects are repressed through the activation of MAPK and PI-3K signaling in mammary epithelial and breast cancer cells (22). Another study indicated that erufosine suppresses breast cancer in vitro and in vivo via its activity on PI-3K, c-Raf and Akt proteins. This study indicates that erufosine possesses high antineoplastic activity not only in breast cancer cell lines in vitro but also in rat mammary carcinoma in vivo. In addition, this may indicate that the mechanism of action of erufosine involves influence on both PI-3K/Akt and Ras/Raf/MAPK signaling pathways (23). Aromatic-turmerone was found to suppress the TPA-induced upregulation of MMP-9 and COX-2 expression by blocking NF- $\mathrm{KB}, \mathrm{PI}-3 \mathrm{~K} / \mathrm{Akt}$ and ERK1/2 signaling in human breast cancer cells. Aromatic-turmerone significantly inhibited TPA-induced invasion, migration and colony formation in human breast cancer cells (24). Therefore, in the present study, we aimed to investigate the regulation by resveratrol of both MMP-9 expression and cell migratory abilities, using HT1080 human fibrosarcoma cells as a model. We also investigated the underlying signaling pathways involved in the molecular mechanism of resveratrol on MMP-9 and cell migration in HT1080 cells. We observed that resveratrol induces MMP-9 expression and cell migration through the p38 kinase and PI-3K pathways in HT1080 human fibrosarcoma cells.

\section{Materials and methods}

Materials. Resveratrol was purchased from Sigma-Aldrich (St. Louis, MO, USA). RPMI-1640 medium and fetal bovine serum (FBS) were purchased from Invitrogen (Burlington, ON, Canada). Streptomycin and penicillin were obtained from Sigma-Aldrich. SB203580 and LY294002 were purchased from Calbiochem (San Diego, CA, USA). MMP-9 (Santa Cruz Biotechnology, Inc., Santa Cruz, CA, USA), pp38 (Cell Signaling Technology, Danvers, MA, USA), p38 (Santa Cruz Biotechnology, Inc.), pAkt (Cell Signaling Technology), Akt and actin antibodies (both from Santa Cruz Biotechnology, Inc.) were used for the experiment.

Cell line and culture. The human fibrosarcoma cell line, HT1080, was obtained from the American Type Culture Collection (Rockville, MD, USA). HT1080 cells were maintained in RPMI-1640 medium containing 10\% FBS, $50 \mu \mathrm{g} / \mathrm{ml}$ streptomycin and $50 \mathrm{U} / \mathrm{ml}$ penicillin. Cell cultures were maintained at $37^{\circ} \mathrm{C}$, in a humidified atmosphere of $5 \% \mathrm{CO}_{2}$ in an incubator. HT1080 cells were plated on culture dishes at a density of $1.2 \times 10^{5}$ cells/dish, and cells at $70-80 \%$ confluency were used for treatment.

MTT assay. Cell growth inhibition was evaluated by MTT assay. HT1080 cells were plated at a density of $1.2 \times 10^{5}$ cells/well on a 96-well plate and maintained overnight for attachment. The next day, the medium was replaced. The cells were treated with various concentrations of resveratrol and were allowed to grow for $24 \mathrm{~h}$. Four hours before the completion of incubation, $10 \mu \mathrm{l}$ of MTT reagent I (methylthiazole tetrazolium, $10 \mathrm{mg} / \mathrm{ml}$ ) was added to each well. After incubation, $100 \mu \mathrm{l}$ of MTT reagent II (solubilization buffer, 10\% SDS with $0.01 \mathrm{~N} \mathrm{HCl,} \mathrm{DMSO)} \mathrm{was}$ added to each well, and the cells were incubated overnight at $37^{\circ} \mathrm{C}$ and $5 \% \mathrm{CO}_{2}$. Finally, the color that developed after the reaction was measured at $595 \mathrm{~nm}$ using an ELISA plate reader.

Flow cytometric analysis. Cells were then treated with various concentrations of resveratrol for $24 \mathrm{~h}$. HT1080 cells were untreated (control) or treated with resveratrol in the absence or presence of SB203580 or LY294002 for 24 h. Cells were harvested and washed twice with phosphate-buffered saline (PBS). Cells were then centrifuged at $1,000 \mathrm{rpm}$ for $10 \mathrm{~min}$ and were resuspended in $70 \%$ cold ethanol at $4^{\circ} \mathrm{C}$ overnight. The fixed cells were washed twice with cold PBS, resuspended in a PBS containing RNase A (50 g/ml; Sigma-Aldrich) at $37^{\circ} \mathrm{C}$ for $30 \mathrm{~min}$ and stained with propidium iodide $(50 \mu \mathrm{g} / \mathrm{ml}$; Molecular Probes, Eugene, OR, USA) for $30 \mathrm{~min}$ in the dark. Cell populations undergoing cell cycle arrest were determined using a flow cytometer (Partec, Munich, Germany).

Gelatin zymography. Gelatin zymography was performed in order to determine the effect of resveratrol on the activity of MMPs. HT1080 cells were seeded on 35-mm tissue culture 
A
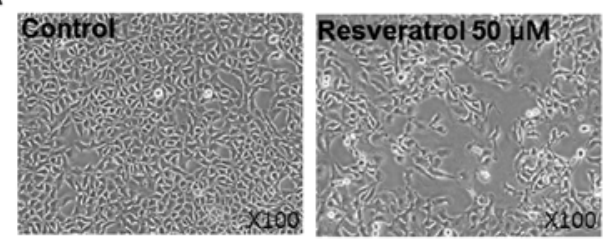

B

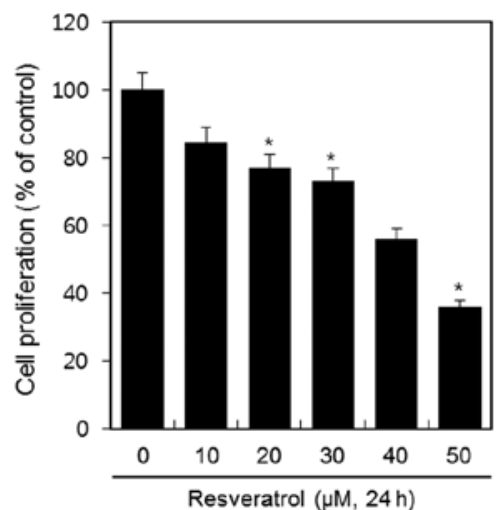

C
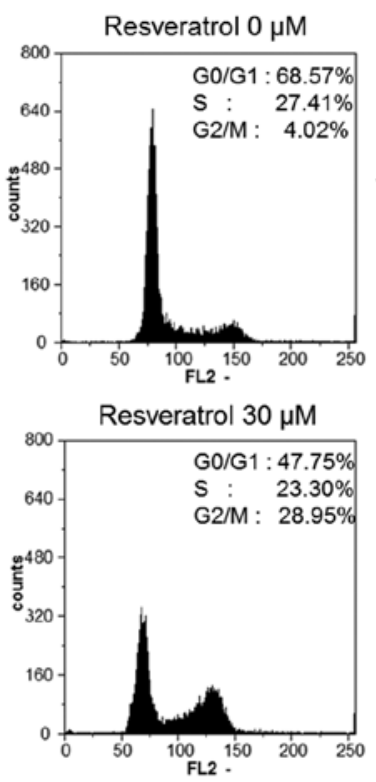

Resveratrol $20 \mu \mathrm{M}$
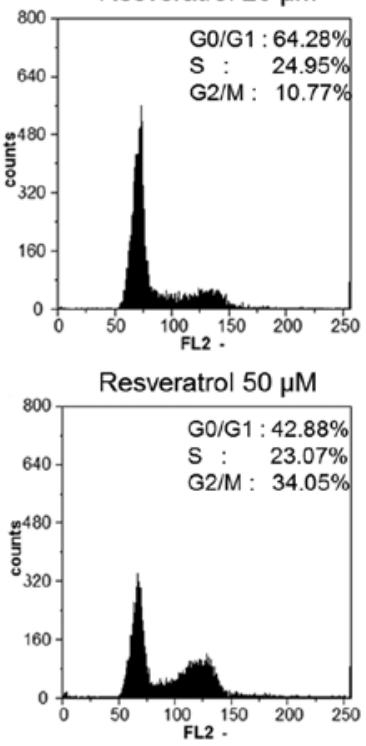

Figure 1. Effects of resveratrol on cell viability and proliferation in HT1080 cells. (A) HT1080 cells were untreated or treated with $50 \mu \mathrm{M}$ of resveratrol for $24 \mathrm{~h}$. Cellular morphology was observed using phase contrast microscopy (magnification, x100). (B) HT1080 cells were treated with the indicated concentrations of resveratrol for $24 \mathrm{~h}$. Cell proliferation was measured using MTT (methylthiazole tetrazolium) assay. $\mathrm{P}<0.05$ compared to the control. (C) Using flow cytometric analysis, we demonstrated the effect of resveratrol on cell cycle arrest in HT1080 cells. Data are presented as the results of a typical experiment and as the means \pm SD.

dishes and maintained overnight for attachment. The next day, the medium was replaced with RPMI containing $2 \%$ serum and cells were incubated overnight. HT1080 cells were treated with resveratrol in the absence or presence of inhibitors (SB203580 and LY294002) in RPMI containing 2\% serum. The supernatants were collected to prepare samples with a loading buffer. Proteins were resolved by $7.5 \%$ SDS-PAGE in the presence of $0.1 \%$ gelatin. After SDS-PAGE, the gels were washed three times with $2.5 \%$ Triton $\mathrm{X}-100$ for $90 \mathrm{~min}$ and incubated with a gelatin incubation buffer $\left(5 \mathrm{mM} \mathrm{CaCl}_{2}, 0.2 \mathrm{M}\right.$ $\mathrm{NaCl}$ and $50 \mathrm{mM}$ Tris) for $24 \mathrm{~h}$. The gels were then stained with Coomassie Blue solution for $1 \mathrm{~h}$ and were exposed to an LAS-3000 imager (Fuji Film Co., Tokyo, Japan). The experimental results were generated as numerical values through the program ImageJ.

Western blot analysis. HT1080 cells grown in 35-mm tissue culture dishes were treated with resveratrol in the absence or presence of inhibitors (SB203580 and LY294002), harvested and washed with cold PBS. Proteins were isolated via cold RIPA lysis buffer (50 mM Tris- $\mathrm{HCl} \mathrm{pH} 7.4,150 \mathrm{mM} \mathrm{NaCl}$, $1 \%$ Nonidet P-40 and $0.1 \%$ SDS), supplemented with protease inhibitors [10 $\mu \mathrm{g} / \mathrm{ml}$ aprotinin, $10 \mu \mathrm{g} / \mathrm{ml}$ leupeptin, $10 \mu \mathrm{g} / \mathrm{ml}$ pepstatin and $1 \mathrm{mM} 4$-(2-aminoethyl) benzenesulfonyl fluoride] and phosphatase inhibitors (1 mM sodium orthovanadate, $1 \mathrm{mM} \mathrm{NaF}$ ) and equal amounts of total cellular proteins were resolved by SDS-PAGE. After SDS-PAGE, the proteins were transferred to the nitrocellulose (NC) membranes (Whatman Schleicher and Schuell, Dachen, Germany). The NC sheet was blocked with 5\% non-fat dry milk in Tris-buffered saline. Antibodies against MMP-9, pp38, p38, pAkt, Akt and actin were used for probing corresponding NC blots overnight at $4^{\circ} \mathrm{C}$. NC membranes were then washed with TBST (Tris- buffered saline/Tween-20) and incubated with horseradish peroxidase-conjugated secondary antibody (Sigma-Aldrich) for $2 \mathrm{~h}$, followed by an exposure using an LAS-3000 imager. The experimental results were generated in numerical values using the program Image $\mathrm{J}$.

Wound healing assay. HT1080 cells were seeded into 35-mm tissue culture dishes and were allowed to attach overnight to $70-80 \%$ confluency in RPMI containing $2 \%$ serum. Cell monolayers were wounded by $1-\mathrm{mm}$ plastic tips and washed twice with PBS in order to remove cell debris. Cells were treated with resveratrol in the absence or presence of inhibitors (SB203580 and LY294002). HT1080 cells migrated into the wound surface, and the number of migrating cells was determined under an inverted microscope.

Data analysis and statistics. The results are expressed as the means \pm SD. Values were calculated from the specified number of determinations. The significance of difference in the experimental and control groups was assessed by one-way ANOVA. A value of $\mathrm{P}<0.05$ was considered to indicate a statistically significant difference.

\section{Results}

Resveratrol inhibits cell viability and proliferation of HT1080 human fibrosarcoma cells. HT1080 cells were treated with or without $50 \mu \mathrm{M}$ of resveratrol for $24 \mathrm{~h}$, and cellular morphology of the cells was analyzed under a microscope. Resveratrol inhibited the growth of HT1080 cells (Fig. 1A). To determine the inhibitory effect of resveratrol on cell viability and proliferation of the HT1080 cells more precisely, we treated the cells with $10,20,30,40$ and $50 \mu \mathrm{M}$ of resveratrol for $24 \mathrm{~h}$ and deter- 
A
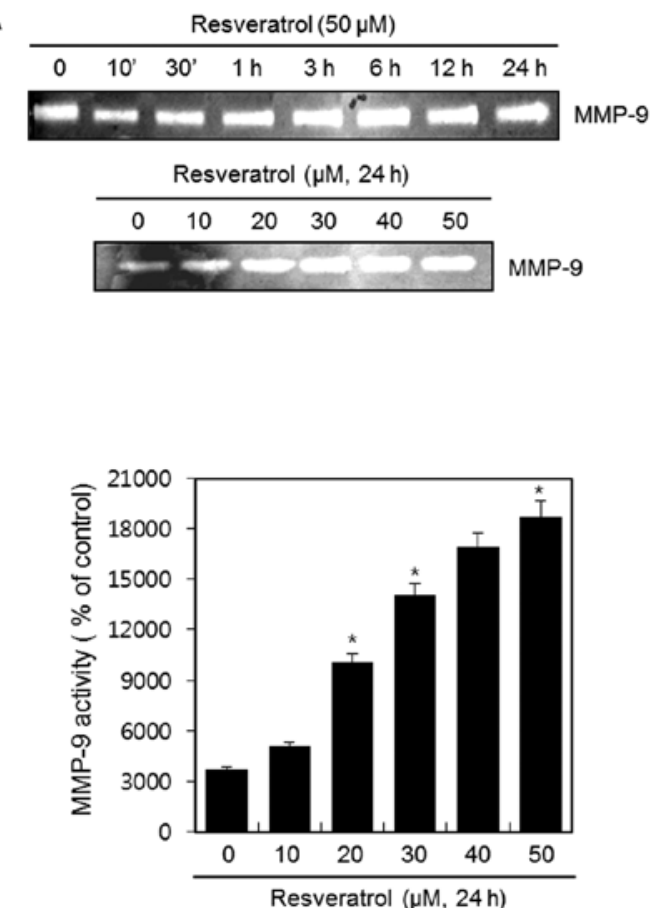

B
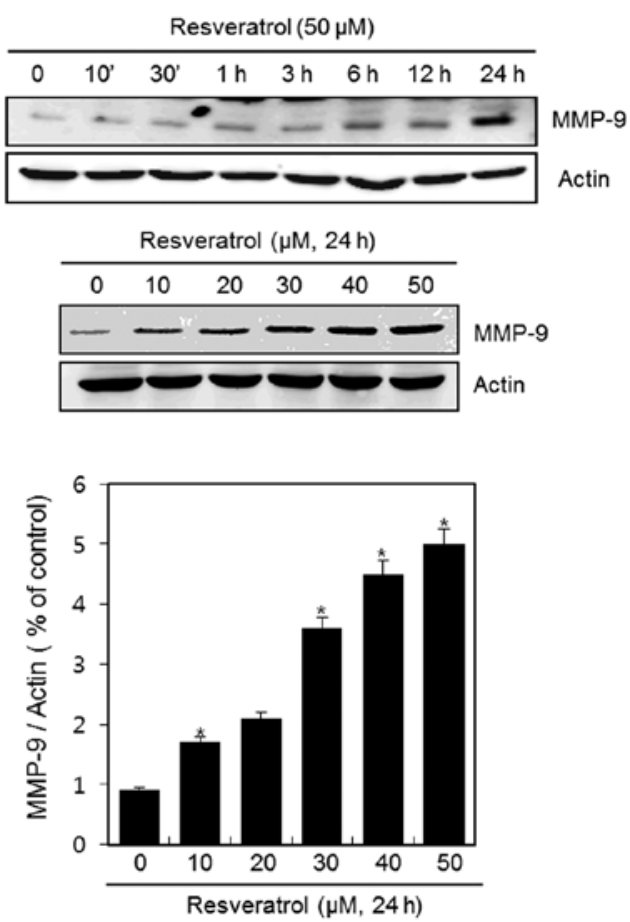

Figure 2. Effects of resveratrol on the activation and expression of MMP-9 in HT1080 cells. (A) HT1080 cells were treated with $50 \mu$ M resveratrol for various time periods or with the indicated concentrations for $24 \mathrm{~h}$. The activation of MMP-9 was detected using a gelatin zymography assay. (B) Expression of MMP-9 was detected using western blot analysis. Actin was used as the loading control. (A and B) The relative amounts of MMP-9 were quantified by densitometric measurement (ImageJ) (bottom graphs). Data are presented as the results of a typical experiment and as the means $\pm \mathrm{SD}$. ${ }^{*} \mathrm{P}<0.05$ compared to the control.

mined the effect using MTT assay. The results demonstrated that cell viability and proliferation decreased as the resveratrol dose increased (Fig. 1B). With $50 \mu \mathrm{M}$ of resveratrol, up to $50 \%$ inhibition of proliferation was observed after a $24-\mathrm{h}$ treatment. Specifically, resveratrol inhibited cell viability and proliferation of HT1080 cells in a dose-dependent manner. Using flow cytometric analysis, we demonstrated the effect of resveratrol on cell cycle arrest in HT1080 cells. HT1080 cells were treated with 20,30 and $50 \mu \mathrm{M}$ of resveratrol for $24 \mathrm{~h}$. We observed that resveratrol induced cell cycle arrest at the $\mathrm{G} 2$ and $\mathrm{M}$ phases. The percentages of cells arrested in the G2/M phase following treatment with resveratrol at concentrations of 20,30 and $50 \mu \mathrm{M}$ were $\sim 10.77,28.95$ and $34.05 \%$, respectively (Fig. 1C).

These results indicated that resveratrol inhibits cell viability and proliferation in HT1080 human fibrosarcoma cells via the induction of G2/M phase cell cycle arrest.

Resveratrol induces the activation and expression of MMP-9 in HT1080 human fibrosarcoma cells. HT1080 cells were untreated (control) or treated with $50 \mu \mathrm{M}$ of resveratrol for the indicated time periods or with various concentrations of resveratrol for $24 \mathrm{~h}$. The activation of MMP-9 was analyzed using gelatin zymography. Resveratrol dramatically induced the activation of MMP-9 in a time- and dose-dependent manner (Fig. 2A). In addition, the expression of MMP-9 was detected using western blot analysis. The expression of MMP-9 was increased after treatment with resveratrol in a time-and dosedependent manner (Fig. 2B). The experimental results were obtained using ImageJ (Fig. 2A and B).
These results suggest that resveratrol induces both the activation and expression of MMP-9 in HT1080 human fibrosarcoma cells.

Resveratrol increases the cell migratory ability of HT1080 human fibrosarcoma cells. We used a wound healing assay in order to evaluate the effect of resveratrol on the cell migration of HT1080 cells. Confluent cell monolayers were scraped and then treated with 20,30 and $50 \mu \mathrm{M}$ of resveratrol for 24 and $48 \mathrm{~h}$. We discovered that resveratrol dramatically increased cell migration of HT1080 cells in a dose-and timedependent manner (Fig. 3A). After $48 \mathrm{~h}$, the wound was almost covered due to the influx of highly migratory cells in the $50-\mu \mathrm{M}$ resveratrol group. In addition, a clear dose-response effect of resveratrol was observed; the percentage of induction of the migratory ability of 20,30 and $50 \mu \mathrm{M}$ of resveratrol was $\sim 50,70$ and $85 \%$, respectively (Fig. 3B).

These results indicate that resveratrol increases the cell migratory ability of HT1080 fibrosarcoma cells in a dose- and time-dependent manner.

Resveratrol induces MMP-9 and cell migratory ability via 338 and Akt kinases in human fibrosarcoma HT1080 cells. The following experiments were performed in order to verify that the upstream signaling pathways of MMP-9 and cell migration were influenced by resveratrol. HT1080 cells were untreated (control) or treated with $50 \mu \mathrm{M}$ of resveratrol for the indicated time periods or with the indicated concentrations of resveratrol for $24 \mathrm{~h}$. The expression of pp38, p38, pAkt, Akt and actin was analyzed using western blot analysis. We observed that 
A

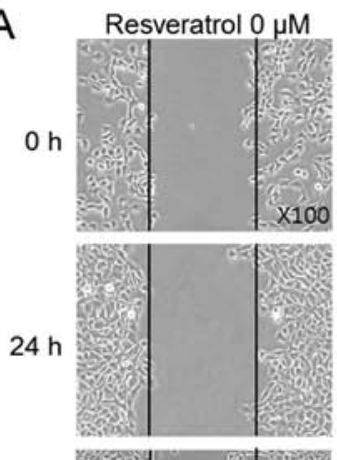

$48 \mathrm{~h}$

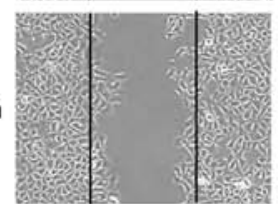

Resveratrol $20 \mu \mathrm{M}$
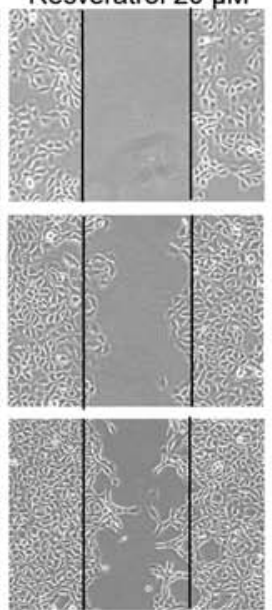

Resveratrol $30 \mu \mathrm{M}$
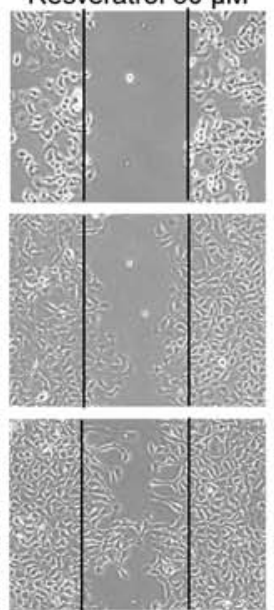

Resveratrol $50 \mu \mathrm{M}$
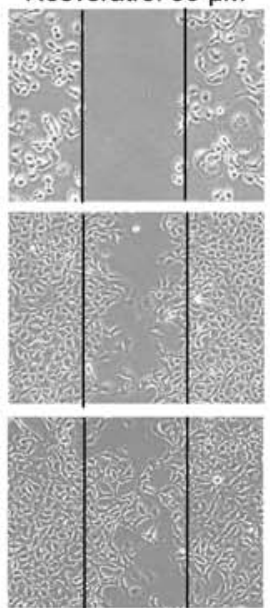

B

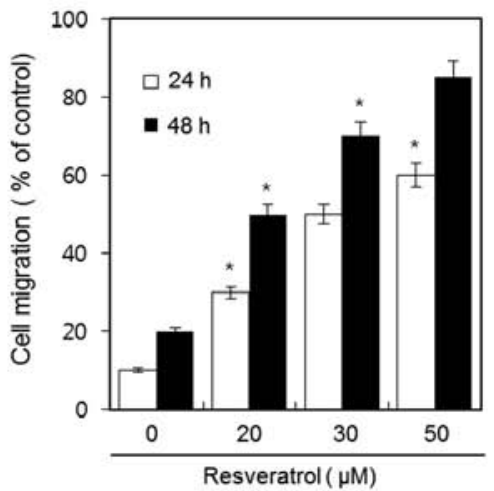

Figure 3. Effects of resveratrol on the cell migratory ability of HT1080 cells. (A) Confluent cell monolayers were scraped and then untreated (control) or treated with 20,30 and $50 \mu \mathrm{M}$ of resveratrol for 24 and $48 \mathrm{~h}$. Cell migratory ability was determined by wound healing assay. (B) Quantification of the wound healing assay. Data are presented as the results of a typical experiment and as the means $\pm \mathrm{SD}$. ${ }^{*} \mathrm{P}<0.05$ compared to the control.

A

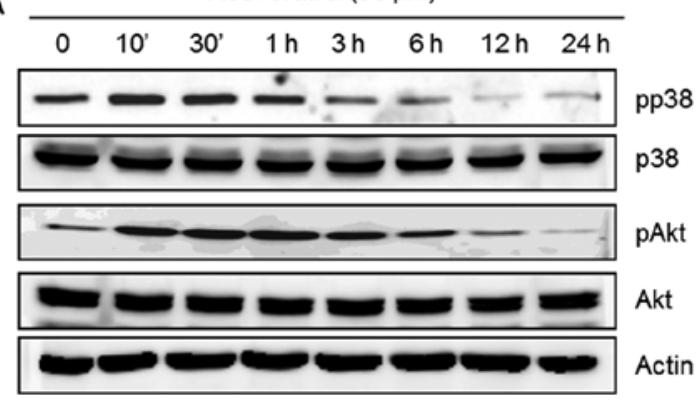

B

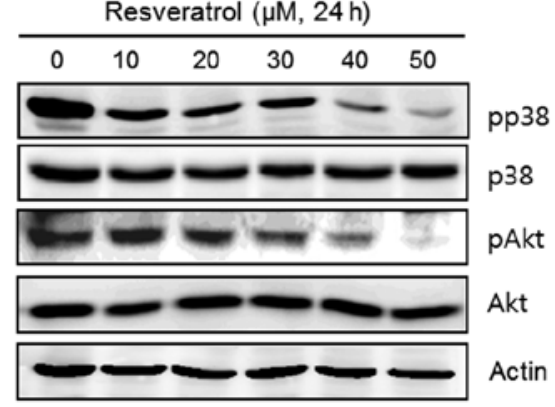

Figure 4. Effects of resveratrol on the activation of p38 and PI-3 kinases in HT1080 cells. (A) HT1080 cells were untreated (control) or treated with $50 \mu \mathrm{M}$ of resveratrol for the indicated time periods. (B) HT1080 cells were untreated (control) or treated with the indicated concentrations of resveratrol for $24 \mathrm{~h}$. The expression of pp38, p38, pAkt, Akt and actin was detected using western blot analysis. p38, Akt and actin were used as loading controls. The data present results of typical experiments from at least three independent experiments.

resveratrol dramatically decreased the phosphorylation of p38 and Akt in a time-dependent manner (Fig. 4A). In addition, the phosphorylation of $\mathrm{p} 38$ and Akt was decreased after treatment with resveratrol in a dose-dependent manner (Fig. 4B).

Next, cells were untreated (control) or treated with resveratrol in the absence or presence of $10 \mu \mathrm{M}$ SB203580 or $10 \mu \mathrm{M}$ LY294002 for $24 \mathrm{~h}$. The activation of MMP-9 was detected using the gelatin zymography assay (Fig. 5A). The expression of pp38, pAkt, MMP-9 and actin was determined by western blot analysis (Fig. 5B). The inhibition of resveratrol-reduced p38 and Akt kinase with SB203580 and LY294002 resulted in a more increased resveratrol-induced activation and expression of MMP-9. The experimental results were obtained using ImageJ (Fig. 5A and B).

The cells were untreated (control) or treated with $50 \mu \mathrm{M}$ of resveratrol in the absence or presence of $10 \mu \mathrm{M} \mathrm{SB} 203580$ or $10 \mu \mathrm{M} \mathrm{LY} 294002$ for $24 \mathrm{~h}$. We investigated whether cell proliferation is involved with cell cycle arrest by flow cytometric analysis. As a result, there was no difference in cell proliferation following single treatment of resveratrol $(50 \mu \mathrm{M})$ when compared with the cells treated in combination with SB203580 or LY294002. Furthermore, treatment with
SB203580 or LY294002 engendered an $\sim 10-13 \%$ apoptosis (Fig. 6A). Confluent cell monolayers were scraped and then used as control, or treated with $50 \mu \mathrm{M}$ resveratrol in the absence or presence of $10 \mu \mathrm{M}$ SB203580 or $10 \mu \mathrm{M}$ LY294002 for 24 and $48 \mathrm{~h}$ (Fig. 6B). Cell migratory ability was detected using a wound healing assay. The inhibition of $\mathrm{p} 38$ and Akt kinases with SB203580 and LY294002 resulted in increased resveratrol-induced cell migration of HT1080 cells. The percentages of induction in cell migration following treatment with $50 \mu \mathrm{M}$ of resveratrol, when compared with treatment with SB203580 and LY294002, was $~ 76$ and 79\% at $24 \mathrm{~h}$, respectively and $\sim 79$ and $82 \%$ at $48 \mathrm{~h}$, respectively (Fig. 6C).

These results suggest that resveratrol induces the activation and expression of MMP-9 and cell migration via p38 kinase and PI-3K in HT1080 human fibrosarcoma cells. Moreover, the results indicate that resveratrol increases cancer metastasis in HT1080 human fibrosarcoma cells.

\section{Discussion}

Invasion is an important step in the growth and metastasis of tumor cells, Metastasis of cancer cells is a complex multistep 
A
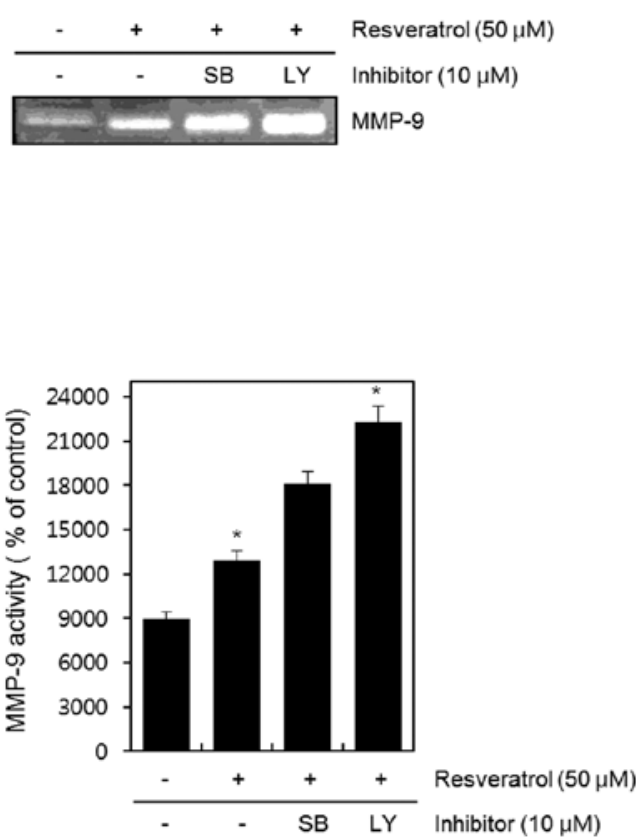

B
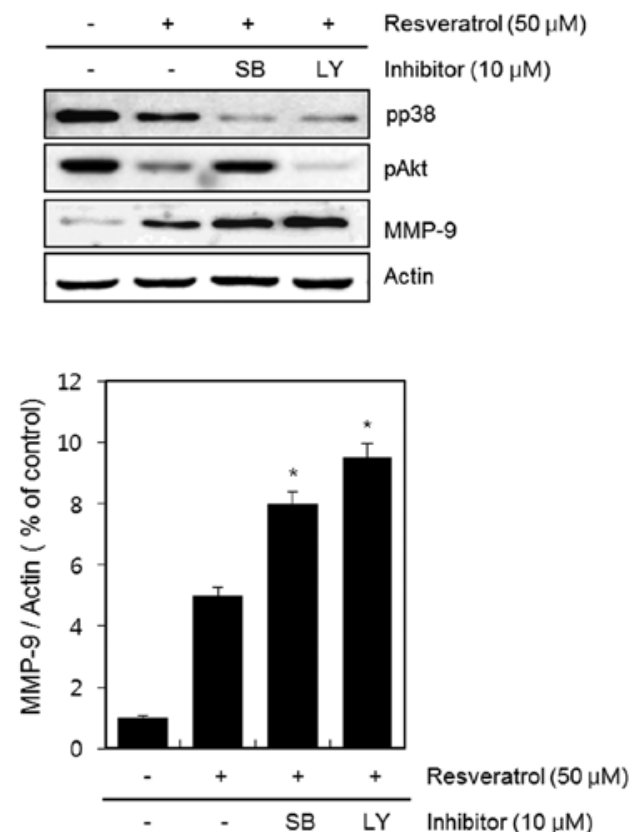

Figure 5. Effects of resveratrol on the regulation of MMP-9 via p38 and PI-3 kinase pathways in HT1080 cells. HT1080 cells were untreated (control) or treated with resveratrol in the absence or presence of $10 \mu \mathrm{M} \mathrm{SB} 203580$ or $10 \mu \mathrm{M} \mathrm{LY} 294002$ for $24 \mathrm{~h}$. (A) Activation of MMP-9 was detected using a gelatin zymography assay. (B) The expression levels of pp38, pAkt, MMP-9 and actin were detected using western blot analysis. Actin was used as the loading control. (A and B) The relative amounts of MMP-9 were quantified by densitometric measurement (ImageJ). Data are presented as the results of a typical experiment and as the means \pm SD. ${ }^{*} \mathrm{P}<0.05$ compared to the control.

A
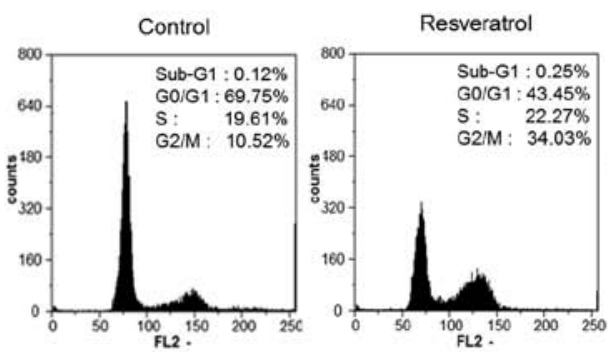

B

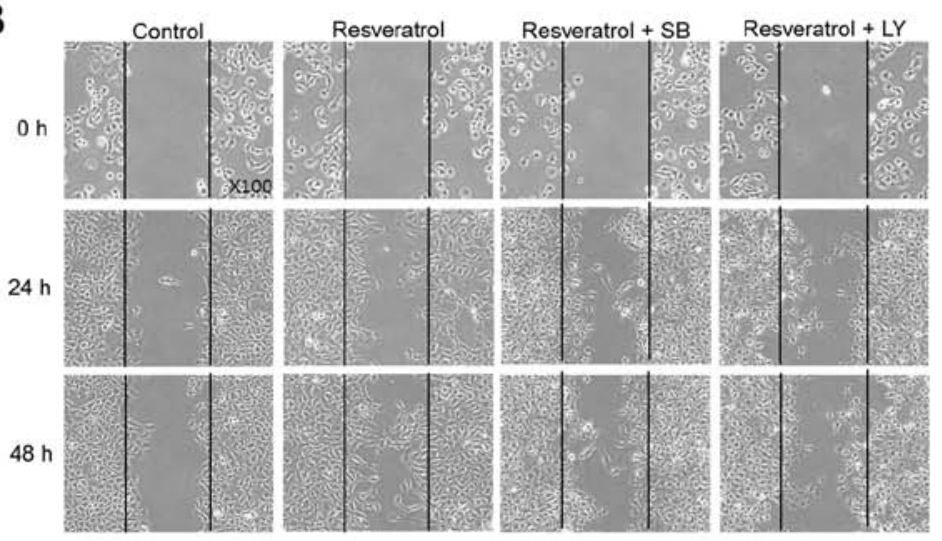

Resveratrol + SB
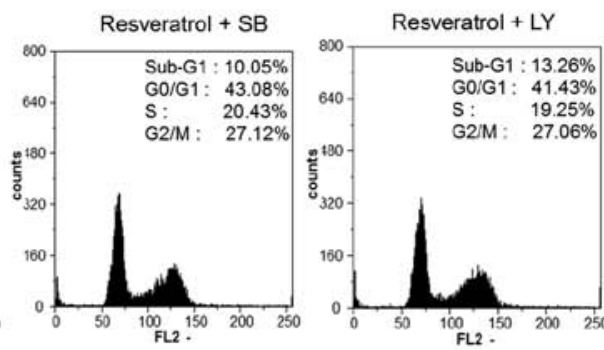

C

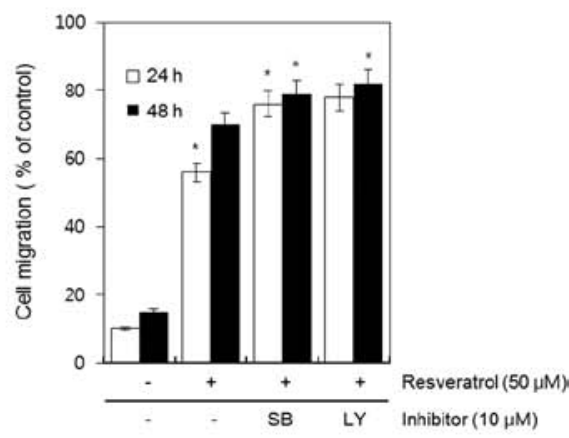

Figure 6. Effects of resveratrol on the regulation of cell migration via p38 and PI-3 kinase pathways in HT1080 cells. (A) HT1080 cells were untreated (control) or treated with resveratrol in the absence or presence of $10 \mu \mathrm{M} \mathrm{SB} 203580$ or $10 \mu \mathrm{M} \mathrm{LY} 294002$ for $24 \mathrm{~h}$. Cell cycle arrest was determined using flow cytometric analysis. (B) Confluent cell monolayers were scraped and then untreated (control) or treated with resveratrol in the absence or presence of $10 \mu \mathrm{M}$ SB203580 or $10 \mu \mathrm{M} \mathrm{LY} 294002$ for 24 and $48 \mathrm{~h}$. Cell migratory ability was determined by wound healing assay. (C) Quantification of the wound healing assay. Data are presented as results of a typical experiment and as the means \pm SD. ${ }^{*} \mathrm{P}<0.05$ compared to the control.

process involving cell invasion, adhesion, invasion and motility (25). The initial step of tumor cell invasion is characterized by the breakdown of the basement membrane, a process depen- dent on type IV collagen-degrading enzymes (26). Tumor metastasis and invasion are the main causes of morbidity and mortality worldwide. MMPs, a family of zinc-dependent 
endopeptidases, have the ability to degrade extracellular matrix (ECM) components. Therefore, MMPs play a fundamental role in tumor invasion and tissue remodeling (27). MMPs facilitate tumor progression and metastasis (28). The secretion and activation of MMP-2 and MMP-9 have been associated with a high metastatic potential in several human carcinomas including breast, colon, lung and pancreatic carcinomas (29). The induction of MMP-2 and MMP-9 involves multiple signaling cascades, particularly the MAPK pathway (30-32). It has been reported that the activation of the MAPK and PI-3K pathways plays a critical role in the modulation of MMP-9 (33). The activation of ERK1/2 and p38 has been found in other systems to be the mechanism for promoting the production of MMPs, which are important for cell proliferation and invasion. It has also been reported that the PI-3K/Akt and nuclear factor- $\mathrm{\kappa B}(\mathrm{NF}-\mathrm{\kappa B})$ pathways are involved in the regulation of MMP-9 (34). It has been suggested that inhibition of p-ERK1/2 and p-JNK leads to a reduction in tumor cells (35). Recent studies have demonstrated that flavonoid baicalein suppresses adhesion, migration and invasion of MDA-MB-231 human breast cancer cells. Baicalein mediates invasive properties of MDA-MB-231 cells by suppressing the activation and expression of MMP-2 and MMP-9, which are important factors for breast cancer cells, including MDA-MB231. The MAPK/MMP signaling pathway in MDA-MB-231 cells contributes to the underlying mechanism of an invasive potential, which suggests that the MAPK signaling pathway is a potential therapeutic target in highly invasive breast cancer cells (36). Dieckol from Ecklonia cava regulates the invasion of human fibrosarcoma cells and modulates MMP-2 and MMP-9 expression via the NF- $\mathrm{\kappa B}$ pathway. It acts as an inhibitor of MMP-2 and MMP-9 expression levels by the downregulation of the NF- $\kappa$ B pathway without significant influence on AP-1 and MAPK pathways. Furthermore, the modulation of MMP-2 and MMP-9 expression levels may be a reason for the suppression of the invasiveness of the HT1080 cell line (37). Previous studies indicate the inhibitory effects of wogonin on the invasion of human breast carcinoma cells by downregulating the expression and activity of MMP-9. This study illustrated that wogonin may inhibit tumor invasion and metastasis in vitro via decreasing both the endogenous and PMA-induced MMP-9 expression and activation. Furthermore, it demonstrated that the downregulation of MMP-9 is potentially associated with the inhibition of PKC translocation and ERK1/2 protein phosphorylation (38).

Resveratrol has been demonstrated to possess anti-cancer, anti-aging, anti-inflammatory and neuroprotective activities. Resveratrol has been shown to inhibit migration and invasion of human breast cancer cells (39). Resveratrol was previously reported to protect cells from oxidative DNA damage caused by hydrogen peroxide and peroxynitrite (40). Recent studies have demonstrated that resveratrol inhibits human lung adenocarcinoma cell metastasis by suppressing the heme oxygenase 1-mediated (HO-1) NF-kB pathway and subsequently, downregulating the expression of MMPs. This study revealed that resveratrol inhibited the expression of MMP-2 and MMP-9 and reduced human lung adenocarcinoma cell metastasis by suppressing HO-1. Moreover, HO-1 inhibition or silencing induced the downregulation of MMP-2 and MMP-9, which was due to the inhibition of the NF- $\mathrm{KB}$-dependent signaling pathway. These findings suggest that resveratrol may act as a therapeutic agent in the inhibition of cancer progression and provide a novel mechanistic insight into the potential effects of resveratrol on the inhibition of tumor invasion and metastasis (41). In a recent study, resveratrol via anti-oxidant activity inhibited MMP via modulation of SIRT1 in human fibrosarcoma cells. The results suggest that the activation of SIRT1 in the presence of resveratrol inhibits the expression of MMP-9 in human fibrosarcoma cells through the suppression of ROS and AP-1 as well as NF- $\kappa$ B activation by the enhanced activity of SIRT1 (42). Resveratrol was found to inhibit tumor cell adhesion to endothelial cells by blocking ICAM-1 expression. Resveratrol blocks ICAM-1 expression and cell adhesion between HT1080 and ECV304 cells, which suggests that the downregulation of ICAM-1 by resveratrol is involved in reducing cell adhesion (43). Moreover, resveratrol has been demonstrated to modulate MED28 (magicin/EG-1) expression and inhibit epidermal growth factor (EGF)-induced migration in MDA-MB-231 human breast cancer cells. The role of MED28 in cell migration reinforces the importance of this multifaceted protein and raises the possibility of clinical application in a combination therapy of resveratrol and MED28 suppression in breast cancer cells (44). Another recent study revealed the effects of resveratrol on cyclooxygenase- 1 and -2 , NF- $\kappa B$, MMP-9 and sirtuin 1 mRNA expression in the hearts of streptozotocin-induced diabetic rats (45). It reported the anti-invasive effect of resveratrol and related methoxy analogues on human hepatocarcinoma cells. Resveratrol may be used to further examine their signal transduction pathways on MMP-9 suppression and TIMP-2 induction for the prevention of hepatoma invasion or metastasis (46). Resveratrol was found to induce gastric cancer cell apoptosis via ROS, independently of sirtuin 1 (47). In addition, it has been reported that resveratrol arrests the cell cycle and induces apoptosis in human hepatocellular carcinoma cells (48).

However, the effect of resveratrol on MMP-9 and the migratory ability in HT1080 human fibrosarcoma cells remains unclear. Therefore, in the present study we investigated the effect of resveratrol on MMP-9 and cell migration in HT1080 cells. In the present study, we discovered that resveratrol at various concentrations inhibited the HT1080 cell viability using MTT assay and FACS analysis (Fig. 1). However, resveratrol dramatically increased the activation and expression of MMP-9 in a dose- and time-dependent manner as determined by gelatin zymography assay and western blot analysis (Fig. 2). We found that resveratrol induced the migratory ability of HT1080 cells as determined by a wound healing assay. Resveratrol induced the migration of HT1080 cells in a dose- and time-dependent manner (Fig. 3). Phosphorylation of p38 and Akt kinases was inhibited by resveratrol in the HT1080 cells in a dose- and time-dependent manner (Fig. 4). To confirm the importance of the p38 and Akt kinases in the regulatory pathway of resveratrol-induced MMP-9 and cell migration, we treated cells with SB203580 and LY294002. The inhibition of p38 and Akt kinases with SB203580 and LY294002 further increased resveratrol-induced MMP-9 activation and expression (Fig. 5). In addition, the suppression of p38 and Akt kinases with SB203580 and LY294002 further increased the resveratrol-induced migratory ability of HT1080 cells (Fig. 6). 
In conclusion, our data indicated that resveratrol inhibited cell proliferation. However, resveratrol also increased the activity and expression of MMP-9 and the migratory ability in HT1080 cells. In addition, resveratrol decreased the phosphorylation of p38 and Akt. Inhibition of p38 and Akt kinases further enhanced the resveratrol-induced MMP-9 activity and expression and cell migration. Our results suggest that resveratrol regulates MMP-9 and cell migratory ability through the p38 kinase and PI-3K pathways in human fibrosarcoma HT1080 cells. Furthermore, resveratrol enhances cancer metastasis in HT1080 human fibrosarcoma cells.

\section{Acknowledgements}

This study was supported by a grant from the National Research Foundation of Korea (NRF) and funded by the Korean Government (MEST) (2011-0027473 and 20120004359).

\section{References}

1. Brisdelli F, D'Andrea G and Bozzi A: Resveratrol: a natural polyphenol with multiple chemopreventive properties. Curr Drug Metab 10: 530-546, 2009

2. Cao Y, Cao R and Brakenhielm E: Antiangiogenic mechanisms of diet-derived polyphenols. J Nutr Biochem 13: 380-390, 2002.

3. Brakenhielm E, Cao R and Cao Y: Suppression of angiogenesis tumor growth, and wound healing by resveratrol, a natura compound in red wine and grapes. FASEB J 15: 1798-1800, 2001

4. Aquilano K, Baldelli S, Rotilio G and Ciriolo MR: TransResveratrol inhibits $\mathrm{H}_{2} \mathrm{O}_{2}$-induced adenocarcinoma gastric cell proliferation via inactivation of MEK1/2-ERK1/2-c-jun signalling axis. Biochem Pharmacol 77: 337-347, 2009.

5. Kozuki Y, Miura Y and Yagasaki K: Resveratrol suppresses hepatoma cell invasion independently of its anti-proliferative action. Cancer Lett 167: 151-156, 2001.

6. Woo JH, Lim JH, Kim YH, et al: Resveratrol inhibits phorbol myristate acetate-induced matrix metalloproteinase-9 expression by inhibiting JNK and PKC delta signal transduction. Oncogene 23: 1845-1853, 2004.

7. Sun C, Hu Y, Liu X, et al: Resveratrol downregulates the constitutional activation of nuclear factor-kappaB in multiple myeloma cells, leading to suppression of proliferation and invasion, arrest of cell cycle, and induction of apoptosis. Cancer Genet Cytogenet 165: 9-19, 2006.

8. Klinge CM, Blankenship KA, Risinger KE, et al: Resveratrol and estradiol rapidly activate MAPK signaling through estrogen receptors alpha and beta in endothelial cells. J Biol Chem 280 7460-7468, 2005.

9. Aziz MH, Kumar R and Ahmad N: Cancer chemoprevention by resveratrol: in vitro and in vivo studies and the underlying mechanisms (Review). Int J Oncol 23: 17-28, 2003.

10. Tang FY, Chiang EP and Sun YC: Resveratrol inhibits heregulinbeta1-mediated matrix metalloproteinase-9 expression and cell invasion in human breast cancer cells. J Nutr Biochem 19: 287-294, 2008.

11. Yilmaz M, Christofori G and Lehembre F: Distinct mechanisms of tumor invasion and metastasis. Trends Mol Med 13: 535-541, 2007.

12. Woessner JF Jr: Matrix metalloproteinases and their inhibitors in connective tissue remodeling. FASEB J 5: 2145-2154, 1991.

13. Hidalgo $M$ and Eckhardt SG: Development of matrix metalloproteinase inhibitors in cancer therapy. J Natl Cancer Inst 93: 178-193, 2001

14. Roach DM, Fitridge RA, Laws PE, Millard SH, Varelias A and Cowled PA: Up-regulation of MMP-2 and MMP-9 leads to degradation of type IV collagen during skeletal muscle reperfusion injury; protection by the MMP inhibitor, doxycycline. Eur J Vasc Endovasc Surg 23: 260-269, 2002.

15. Bachmeier BE, Iancu CM, Jochum $M$ and Nerlich AG: Matrix metalloproteinases in cancer: comparison of known and novel aspects of their inhibition as a therapeutic approach. Expert Rev Anticancer Ther 5: 149-163, 2005.
16. Lee SO, Jeong YJ, Im HG, Kim $\mathrm{CH}$, Chang $\mathrm{YC}$ and Lee IS Silibinin suppresses PMA-induced MMP-9 expression by blocking the AP-1 activation via MAPK signaling pathways in MCF-7 human breast carcinoma cells. Biochem Biophys Res Commun 354: 165-171, 2007.

17. Dhillon AS, Hagan S, Rath O and Kolch W: MAP kinase signalling pathways in cancer. Oncogene 26: 3279-3290, 2007.

18. Bradham C and McClay DR: p38 MAPK in development and cancer. Cell Cycle 5: 824-828, 2006.

19. Lee SJ, Kim WJ and Moon SK: Role of the p38 MAPK signaling pathway in mediating interleukin-28A-induced migration of UMUC-3 cells. Int J Mol Med 30: 945-952, 2012.

20. Zhang B, Wang X, Cai F, Chen W, Loesch U, Bitzer J and Zhong XY: Effects of salinomycin on human ovarian cancer cell line OV2008 are associated with modulating p38 MAPK. Tumour Biol: July 7, 2012 (Epub ahead of print).

21. Foster FM, Traer CJ, Abraham SM and Fry MJ: The phosphoinositide (PI) 3-kinase family. J Cell Sci 116: 3037-3040, 2003.

22. Cotrim CZ, Fabris V, Doria ML, et al: Estrogen receptor beta growth-inhibitory effects are repressed through activation of MAPK and PI3K signalling in mammary epithelial and breast cancer cells. Oncogene: July 2, 2012 (Epub ahead of print).

23. Dineva IK, Zaharieva MM, Konstantinov SM, Eibl H and Berger MR: Erufosine suppresses breast cancer in vitro and in vivo for its activity on PI3K, c-Raf and Akt proteins. J Cancer Res Clin Oncol: June 30, 2012 (Epub ahead of print).

24. Park SY, Kim YH, Kim YH and Lee SJ: Aromatic-turmerone attenuates invasion and expression of MMP-9 and COX-2 through inhibition of NF- $\mathrm{B}$ activation in TPA-induced breast cancer cells. J Cell Biochem: June 27, 2012 (Epub ahead of print).

25. Nam KS and Shon YH: Suppression of metastasis of human breast cancer cells by chitosan oligosaccharides. J Microbiol Biotechnol 19: 629-633, 2009.

26. Lu N, Ling Y, Gao Y, et al: Endostar suppresses invasion through downregulating the expression of matrix metalloproteinase-2/9 in MDA-MB-435 human breast cancer cells. Exp Biol Med (Maywood) 233: 1013-1020, 2008.

27. Sato H, Takino T, Okada Y, Cao J, Shinagawa A, Yamamoto E and Seiki M: A matrix metalloproteinase expressed on the surface of invasive tumour cells. Nature 370: 61-65, 1994.

28. Khokha R: Suppression of the tumorigenic and metastatic abilities of murine B16-F10 melanoma cells in vivo by the overexpression of the tissue inhibitor of the metalloproteinases-1. J Natl Cancer Inst 86: 299-304, 1994.

29. Rosenblum G, Meroueh SO, Kleifeld O, et al: Structural basis for potent slow binding inhibition of human matrix metalloproteinase-2 (MMP-2). J Biol Chem 278: 27009-27015, 2003.

30. Kim ES, Sohn YW and Moon A: TGF-beta-induced transcriptional activation of MMP-2 is mediated by activating transcription factor (ATF) 2 in human breast epithelial cells. Cancer Lett 252: 147-156, 2007.

31. Cohen M, Meisser A, Haenggeli L and Bischof P: Involvement of MAPK pathway in TNF-alpha-induced MMP-9 expression in human trophoblastic cells. Mol Hum Reprod 12: 225-232, 2006.

32. Kajanne R, Miettinen P, Mehlem A, et al: EGF-R regulates MMP function in fibroblasts through MAPK and AP-1 pathways. J Cell Physiol 212: 489-497, 2007.

33. Westermarck J, Holmström T, Ahonen M, et al: Enhancement of fibroblast collagenase-1 (MMP-1) gene expression by tumor promoter okadaic acid is mediated by stress-activated protein kinases Jun N-terminal kinase and p38. Matrix Biol 17: 547-557, 1998.

34. Cho HJ, Kang JH, Kwak JY, et al: Ascofuranone suppresses PMA-mediated matrix metalloproteinase-9 gene activation through the Ras/Raf/MEK/ERK- and Ap1-dependent mechanisms. Carcinogenesis 28: 1104-1110, 2007.

35. Hong IK, Kim YM, Jeoung DI, Kim KC and Lee H: Tetraspanin CD9 induces MMP-2 expression by activating p38 MAPK, JNK and c-Jun pathways in human melanoma cells. Exp Mol Med 37: 230-239, 2005.

36. Wang L, Ling Y, Chen Y, et al: Flavonoid baicalein suppresses adhesion, migration and invasion of MDA-MB-231 human breast cancer cells. Cancer Lett 297: 42-48, 2010.

37. Zhang C, Li Y, Qian ZJ, Lee SH, Li YX and Kim SK: Dieckol from Ecklonia cava regulates invasion of human fibrosarcoma cells and modulates MMP-2 and MMP-9 expression via NF- $\kappa$ B pathway. Evid Based Complement Alternat Med 2011: 140462, 2011.

38. Chen P, Lu N, Ling Y, et al: Inhibitory effects of wogonin on the invasion of human breast carcinoma cells by downregulating the expression and activity of matrix metalloproteinase- 9 . Toxicology 282: 122-128, 2011. 
39. Tang FY, Su YC, Chen NC, Hsieh HS and Chen KS: Resveratrol inhibits migration and invasion of human breast-cancer cells. Mol Nutr Food Res 52: 683-691, 2008.

40. Johnson MK and Loo G: Effects of epigallocatechin gallate and quercetin on oxidative damage to cellular DNA. Mutat Res 459: 211-218, 2000

41. Liu PL, Tsai JR, Charles AL, et al: Resveratrol inhibits human lung adenocarcinoma cell metastasis by suppressing heme oxygenase 1-mediated nuclear factor-kappaB pathway and subsequently downregulating expression of matrix metalloproteinases. Mol Nutr Food Res 54 (Suppl 2): S196-S204, 2010.

42. Lee SJ and Kim MM: Resveratrol with antioxidant activity inhibits matrix metalloproteinase via modulation of SIRT1 in human fibrosarcoma cells. Life Sci 88: 465-472, 2011.

43. Park JS, Kim KM, Kim MH, et al: Resveratrol inhibits tumor cell adhesion to endothelial cells by blocking ICAM-1 expression. Anticancer Res 29: 355-362, 2009.

44. Lee MF, Pan MH, Chiou YS, Cheng AC and Huang $\mathrm{H}$ : Resveratrol modulates MED28 (Magicin/EG-1) expression and inhibits epidermal growth factor (EGF)-induced migration in MDA-MB-231 human breast cancer cells. J Agric Food Chem 59: 11853-11861, 2011.
45. Yar AS, Menevse S and Alp E: The effects of resveratrol on cyclooxygenase-1 and -2, nuclear factor kappa beta, matrix metalloproteinase-9, and sirtuin 1 mRNA expression in hearts of streptozotocin-induced diabetic rats. Genet Mol Res 10: 2962-2975, 2011.

46. Weng $\mathrm{CJ}, \mathrm{Wu} \mathrm{CF}$, Huang $\mathrm{HW}, \mathrm{Wu} \mathrm{CH}, \mathrm{Ho} \mathrm{CT}$ and Yen GC: Evaluation of anti-invasion effect of resveratrol and related methoxy analogues on human hepatocarcinoma cells. J Agric Food Chem 58: 2886-2894, 2010.

47. Liao PC, Ng LT, Lin LT, Richardson CD, Wang GH and Lin CC: Resveratrol arrests cell cycle and induces apoptosis in human hepatocellular carcinoma Huh-7 cells. J Med Food 13: 1415-1423, 2010.

48. Wang Z, Li W, Meng X and Jia B: Resveratrol induces gastric cancer cell apoptosis via reactive oxygen species, but independent of sirtuin1. Clin Exp Pharmacol Physiol 39: 227-232, 2012. 